\title{
SEMI-ARITHMETIC FUCHSIAN GROUPS AND MODULAR EMBEDDINGS
}

\author{
PAUL SCHMUTZ SCHALLER AND JÜRGEN WOLFART
}

\section{Introduction}

Arithmetic Fuchsian groups are the most interesting and most important Fuchsian groups owing to their significance for number theory and owing to their geometric properties. However, for a fixed signature there exist only finitely many nonconjugate arithmetic Fuchsian groups; it is therefore desirable to extend this class of Fuchsian groups. This is the motivation of our definition of semi-arithmetic Fuchsian groups. Such a group may be defined as follows (for the precise formulation see Section 2). Let $\Gamma$ be a cofinite Fuchsian group and let $\Gamma^{2}$ be the subgroup generated by the squares of the elements of $\Gamma$. Then $\Gamma$ is semi-arithmetic if $\Gamma$ is contained in an arithmetic group $\Delta$ acting on a product $\mathrm{H}^{r}$ of upper halfplanes. Equivalently, $\Gamma$ is semi-arithmetic if all traces of elements of $\Gamma^{2}$ are algebraic integers of a totally real field. Well-known examples of semi-arithmetic Fuchsian groups are the triangle groups (and their subgroups of finite index) which are almost all non-arithmetic with the exception of 85 triangle groups listed by Takeuchi [16].

While it is still an open question as to what extent the non-arithmetic Fuchsian triangle groups share the geometric properties of arithmetic groups, it is a fact that their automorphic forms share certain arithmetic properties with modular forms for arithmetic groups. This has been clarified by Cohen and Wolfart [5] who proved that every Fuchsian triangle group $\Gamma$ admits a modular embedding, meaning that there exists an arithmetic group $\Delta$ acting on $\mathrm{H}^{r}$, a natural group inclusion

$$
f: \Gamma \longrightarrow \Delta
$$

and a compatible holomorphic embedding

that is with

$$
F: \mathrm{H} \longrightarrow \mathrm{H}^{r}
$$

for all $\gamma \in \Gamma$ and all $z \in \mathrm{H}$.

$$
F(\gamma z)=f(\gamma) F(z)
$$

This theorem extends to all finite index subgroups of Fuchsian triangle groups (and of course also holds for all arithmetic Fuchsian groups which are not triangle groups). Modular embeddings are related to transcendence questions (see [5]); for a description of the image of the modular embedding of the triangle group $[2,5, \infty]$ (see Schmidt [12]).

It is a natural question (first asked in [5]) as to whether there is a wider class of semiarithmetic Fuchsian groups admitting a modular embedding. In order to treat this question one should first find more examples of such groups. We therefore construct in Section 3 some new sequences of semi-arithmetic groups showing that there are

Received 26 January 1998; revised 4 June 1998.

2000 Mathematics Subject Classification 11F06, 11F41, 20H10, 30F35.

J. London Math. Soc. (2) 61 (2000) 13-24 
infinitely many non-conjugate semi-arithmetic groups of the same signature; hence they really constitute an extension of the arithmetic groups. However, we will see in Section 4 that in contrast to triangle groups they do not admit modular embeddings. These new semi-arithmetic Fuchsian groups have the following property.

Let $\Gamma$ be a semi-arithmetic Fuchsian group. Let $\phi$ be one of the embeddings of the corresponding number field $k$ (generated by the traces of the elements of $\Gamma^{2}$ ) into $\mathrm{R}$, extendable in a natural way to an embedding of $\Gamma^{2}$ and even of $\Gamma$ into $\operatorname{PSL}(2, R)$. Then (this is the property) there exists an embedding $\phi$ such that $\phi(\Gamma)$ is again a Fuchsian group, but $\phi$ is not a conjugation in $\operatorname{PSL}(2, \mathrm{R})$.

\section{Definition of semi-arithmetic Fuchsian groups}

Definition 1. (i) Denote by $\mathrm{H}$ the upper halfplane. identity

(ii) Let $k$ be a totally real number field of degree $n=[k: 0]$. Denote by $\phi_{1}$ the

$$
\phi_{1}: k \longrightarrow \mathrm{R} \text {. }
$$

Let $B$ be a quaternion algebra over $k$ such that from the embeddings

$$
\phi_{i}: k \longrightarrow \mathrm{R}, \quad i=2, \ldots, n,
$$

$\phi_{r+1}, \phi_{r+2}, \ldots \phi_{n}, r \geqslant 1$, extend to embeddings of $B$ into the Hamilton quaternion algebra while $\phi_{1}, \ldots, \phi_{r}$ extend to embeddings of $B$ into the matrix algebra $M(2, \mathrm{R})$.

Let $O$ be an order of $B$ and put

$$
U:=\{\varepsilon \in O: \varepsilon O=O \text { and } n(\varepsilon)=\operatorname{det} \varepsilon=1\}
$$

where $n(\varepsilon)$ is the reduced norm. Define $\Gamma(B, O):=\phi_{1}(U) \subset \mathrm{SL}(2, \mathrm{R})$. Define the action of $\Gamma(B, O)$ on $\mathrm{H}^{r}$ by components:

$$
\varepsilon:\left(z_{1}, \ldots, z_{r}\right) \longmapsto\left(\phi_{1}(\varepsilon) z_{1}, \ldots, \phi_{r}(\varepsilon) z_{r}\right)
$$

where $z_{i} \longmapsto \phi_{i}(\varepsilon) z_{i}$ is the usual action given by linear fractional transformation, $i=1, \ldots, r$.

(iii) A subgroup of $\operatorname{SL}(2, \mathrm{R})$ is called an arithmetic group acting on $\mathrm{H}^{\mathrm{r}}$ if it is commensurable to a $\Gamma(B, O)$ defined above. By abuse of language, we also call its canonical image $\Delta$ in $\operatorname{PSL}(2, \mathrm{R})$ an arithmetic group acting on $\mathrm{H}^{\mathrm{r}}$, obtained by the identification of each element with its negative. In the particular case $r=1$ we call it an arithmetic Fuchsian group.

Remark 1. (i) By Shimura [14] the above-defined action of $\Gamma(B, O)$ on $\mathrm{H}^{r}$ is discontinuous and of finite covolume. Therefore, in the case $r=1$, an arithmetic group $\Gamma$ is a discrete subgroup of $\operatorname{PSL}(2, \mathrm{R})$. For basic material on arithmetic Fuchsian groups, see for example Katok [8].

(ii) The definition given above is the classical definition of arithmetic Fuchsian groups. Takeuchi [15] proved that the following Definition 2(iv) is equivalent.

Definition 2. (i) For a Fuchsian group $\Gamma$ put

$$
\Gamma^{2}:=\left\langle\gamma^{2}: \gamma \in \Gamma\right\rangle \text {. }
$$

(ii) Let $\Gamma$ be a Fuchsian group. Then

$$
\operatorname{Tr}(\Gamma):=\{|\operatorname{tr}(\gamma)|: \gamma \in \Gamma\}
$$

is called the trace set of $\Gamma(\operatorname{tr}(\gamma)$ is the trace of $\gamma)$. 
(iii) A Fuchsian group $\Gamma$ is called cofinite if the corresponding Riemann surface $\mathrm{H} / \Gamma$ has finite area; it is called cocompact if $\mathrm{H} / \Gamma$ is compact ('cocompact' implies 'cofinite').

(iv) A cofinite Fuchsian group $\Gamma$ is an arithmetic Fuchsian group if and only if the two following conditions hold:

(a) $k=\mathrm{Q}\left(\operatorname{Tr}\left(\Gamma^{2}\right)\right)$ is a number field of finite degree $n=[k: \mathrm{Q}]$ and $\operatorname{Tr}\left(\Gamma^{2}\right)$ is contained in the ring of integers $\mathcal{O}$ of $k$.

(b) Let $\phi_{i}$ be one of the $n$ embeddings of $k$ into $\mathrm{C}$ which is not the identity. Then for all $\gamma \in \Gamma^{2}$

$$
\phi_{i}(|\operatorname{tr}(\gamma)|) \in[-2,2] .
$$

REMARK 2. If $\Gamma$ is finitely generated, for example if $\Gamma$ is cofinite, the subgroup $\Gamma^{2}$ is normal and of finite index in $\Gamma$. By condition (b), $\mathrm{Q}\left(\operatorname{Tr}\left(\Gamma^{2}\right)\right)$ is totally real. In view of this second definition of arithmetic Fuchsian groups the following generalization is natural.

Definition 3. A cofinite Fuchsian group $\Gamma$ is called semi-arithmetic if $k=$ $\mathrm{Q}\left(\operatorname{Tr}\left(\Gamma^{2}\right)\right)$ is a totally real number field of finite degree $n=[k: \mathrm{Q}]$ and $\operatorname{Tr}\left(\Gamma^{2}\right)$ is contained in the ring of integers $\mathcal{O}$ of $k$. $\Gamma$ is called strictly semi-arithmetic if $\Gamma$ is not an arithmetic Fuchsian group.

Proposition 1. Let $\Gamma$ be a cofinite Fuchsian group. Then the following two conditions are equivalent.

(i) $\Gamma$ is semi-arithmetic.

(ii) $\Gamma$ is commensurable to a subgroup $S$ of an arithmetic group $\Delta$ acting on $\mathrm{H}^{r}$.

Proof. (ii) $\Rightarrow$ (i). By assumption (ii), the group $\Gamma$ is commensurable with $S$ where $S \subset \Delta \subset \operatorname{PSL}(2, \mathrm{R})$ and the preimage $\bar{\Delta}$ of $\Delta$ in $\operatorname{SL}(2, \mathrm{R})$ is commensurable with some $\Gamma(B, O)$. Then by Borel [2], $\Delta^{2}$ is contained in the image of $\Gamma(B, O)$ in $\operatorname{PSL}(2, \mathrm{R})$; thus $\operatorname{Tr}\left(S^{2}\right)$ consists of integers in the totally real number field $\mathrm{Q}\left(\operatorname{Tr}\left(S^{2}\right)\right)$. This field does not change if we replace $S^{2}$ by the commensurable group $\Gamma^{2}$; see Reid [11] or Hilden, Lozano and Montesinos-Amilibia [7, Lemma 1.12]. Using the eigenvalues it is easy to see that the integrality of the traces is also preserved under the passage to a commensurable group.

(i) $\Rightarrow$ (ii). By [15], $S:=\Gamma^{2}$ (more precisely its preimage in $\operatorname{SL}(2, \mathrm{R})$ ) generates an order

of a quaternion algebra

$$
O=\left\{\sum a_{i} \gamma_{i}: a_{i} \in \mathcal{O}, \gamma_{i} \in \Gamma^{2}\right\}
$$

$$
B=\left\{\sum a_{i} \gamma_{i}: a_{i} \in k, \gamma_{i} \in \Gamma^{2}\right\}
$$

over $k$ as an $\mathcal{\theta}$-submodule of $M(2, \mathrm{R})$ such that $S$ is contained in the units of $O$ of reduced norm 1 . We thus can take $\Delta:=\Gamma(B, O) /\{ \pm 1\}$.

We call this group $\Delta$ the arithmetic group generated by $\Gamma^{2}$. Then we can distinguish arithmetic Fuchsian groups from strictly semi-arithmetic ones as follows.

COROLlary 1. Let $\Gamma$ be a semi-arithmetic Fuchsian group and $\Delta$ be the arithmetic group generated by $\Gamma^{2}$. Then $\Gamma$ is strictly semi-arithmetic if and only if $\Gamma^{2}$ has infinite index in $\Delta$. 
Proof. By the invariance of the trace field $k$ under commensurability $[7,11]$, the quaternion algebra $B$ is also invariant (up to isomorphism) and hence so is the number $r$ of its unramified infinite places. Recall that $\Gamma$ and $\Delta$ act as cofinite discontinuous groups on $\mathrm{H}$ and $\mathrm{H}^{r}$, respectively. Therefore, $\Gamma^{2}$ has finite index in $\Delta$ if $r=1$; if $r>1$ the Fuchsian group $\Gamma^{2}$ does not act as cofinite group on $\mathrm{H}^{r}$ whence it cannot have finite index in $\Delta$, and this alternative depends only on the commensurability class of $\Gamma$.

Remark 3. (i) Condition (ii) of Proposition 1 can therefore also be used as a definition of semi-arithmetic Fuchsian groups, now given in the language of the classical definition of arithmetic Fuchsian groups.

(ii) Not only $\Gamma^{2}$, but also $\Gamma$ itself, can be considered as a subgroup of a finite extension of $\Delta$ (thus all embeddings $\phi_{i}$ of $k$ into $\mathrm{R}$ may be extended also to embeddings of $\Gamma$ into $\operatorname{PSL}(2, R)$ or the Hamiltonians) for the following reason. Since $\Gamma^{2}$ is normal in $\Gamma$, every $\gamma \in \Gamma$ (more precisely its preimage in $\mathrm{SL}(2, \mathrm{R}))$ defines an automorphism

$$
\alpha_{\gamma}: B \longrightarrow B: \sum a_{i} \gamma_{i} \longmapsto \sum a_{i} \gamma \gamma_{i} \gamma^{-1}
$$

of the quaternion algebra $B$ defined in the proof of Proposition 1. By the Skolem-Noether theorem $\alpha_{\gamma}$ is an inner automorphism of $B$, meaning that $\alpha_{\gamma}(x)=$ $a x a^{-1}$ for every $x \in B$ and a fixed $a \in B$. Now $B \subset M(2, \mathrm{R})$, so $\gamma^{-1} a$ lies in the centre of $M(2, \mathrm{R})$. Thus, up to a scalar, $\gamma$ lies in $B$, and $\Gamma$ is the $\operatorname{PSL}(2, \mathrm{R})$-image of a subgroup of the normalizer $N(O)$ of $O$ in $B$. By [2] again, $\Gamma$ is therefore contained in a finite extension of $\Delta$. We thank the referee for providing us with this argument.

We now define a particularly interesting subclass of semi-arithmetic Fuchsian groups.

Definition 4. Let $\Gamma$ be a cofinite Fuchsian group such that there exists a natural group inclusion

$$
f: \Gamma \longrightarrow \Delta
$$

into an arithmetic group $\Delta$ acting on $\mathrm{H}^{r}, r \geqslant 1$, and a compatible holomorphic embedding

that is with

$$
F: \mathrm{H} \longrightarrow \mathrm{H}^{r},
$$

$$
F(\gamma z)=f(\gamma) F(z)
$$

for all $\gamma \in \Gamma$ and all $z \in \mathrm{H}$. Then $(f, F)$ is called a modular embedding and we say that $\Gamma$ admits a modular embedding.

Lemma 1. Let $\Gamma$ be a cofinite Fuchsian group which admits a modular embedding. Then $\Gamma$ is semi-arithmetic.

Proof. Otherwise $\Gamma$ cannot be included into an arithmetic group $\Delta$ acting on $\mathrm{H}^{r}$.

Remark 4. Let $\Gamma$ be a semi-arithmetic group, let $k=\mathrm{Q}\left(\operatorname{Tr}\left(\Gamma^{2}\right)\right)$ be the corresponding number field and let $\phi$ be one of the embeddings of $k$ into $\mathrm{R}$. The elements of $\Gamma$ are matrices in $\operatorname{PSL}(2, R)$; more precisely they may be taken in $\operatorname{PSL}\left(2, k^{\prime}\right)$ for some field extension $k^{\prime}$ of $k$ of finite degree (for the possibilities for choosing $k^{\prime}$ see Waterman and Maclachlan [17]). Applying an extension of $\phi$ to these 
matrix entries we obtain another way to define $\phi(\gamma)$ for all $\gamma \in \Gamma$ which turns out to be independent of the choice of $k^{\prime}$ and of the extension of $\phi$ to $k^{\prime}$ (up to a common conjugation in $\operatorname{PSL}(2, R)$ which is sufficient here).

Proposition 2. Let $\Gamma$ be a cofinite Fuchsian group. Let $k=\mathrm{Q}\left(\operatorname{Tr}\left(\Gamma^{2}\right)\right)$. Then the following two conditions are equivalent.

(i) $\Gamma$ admits a modular embedding.

(ii) $\Gamma$ is semi-arithmetic, and for all embeddings

$$
\phi_{j}: k \longrightarrow \mathrm{R}
$$

for which $\phi_{j}\left(\operatorname{Tr}\left(\Gamma^{2}\right)\right)$ is not contained in the interval $[-2,2]$, the functional equation

$$
F_{j}(\gamma z)=\phi_{j}(\gamma) F_{j}(z), \quad \gamma \in \Gamma,
$$

is solvable by a holomorphic function

$$
F_{j}: \mathrm{H} \longrightarrow \mathrm{H} \text {. }
$$

Proof. (i) $\Rightarrow$ (ii). The $F_{j}$ are the components of $F$ in the modular embedding $(f, F)$.

(ii) $\Rightarrow$ (i). Let $\phi_{j_{1}}, \ldots, \phi_{j_{r}}$ be all the embeddings for which $\phi_{j_{i}}\left(\operatorname{Tr}\left(\Gamma^{2}\right)\right)$ is not contained in the interval $[-2,2]$. Since $\phi_{1}$ is the identity, we have $r \geqslant 1$. We may suppose that $\phi_{j_{1}}=\phi_{1}$ and extend it by the identity to $\Gamma \hookrightarrow \operatorname{PSL}(2, R)$. Put

$$
f(\gamma)=\left(\phi_{j_{1}}(\gamma), \ldots, \phi_{j_{r}}(\gamma)\right)
$$

for all $\gamma \in \Gamma$. Then $f$ defines a group inclusion of $\Gamma$ into an arithmetic group $\Delta$ acting on $\mathrm{H}^{r}$, constructed as in the proof of Proposition 1 and Remark 3(ii).

Put $F:=\left(F_{j_{1}}, \ldots, F_{j_{r}}\right)$. Then $(f, F)$ is a modular embedding of $\Gamma$.

Remark 5. We will see in Section 4 that not all semi-arithmetic Fuchsian groups admit a modular embedding. On the other hand, by [5] all Fuchsian triangle groups and their subgroups admit a modular embedding, but only a few of them are arithmetic [16].

We conclude this section with a further characterization of arithmetic Fuchsian groups.

Schmutz [13] has proved that if $\Gamma$ is a cofinite Fuchsian group which is not cocompact, then $\Gamma$ is arithmetic if and only if there exists a constant $C$ such that

$$
\#\{a \in \operatorname{Tr}(\Gamma): a<x\} \leqslant C x, \quad \forall x .
$$

The conjecture is that the theorem also holds in the compact case. An important open question is whether semi-arithmetic Fuchsian groups can be characterized by the growth of the trace set. This problem is related to questions in quantum chaos; see for example Luo and Sarnak [9].

\section{Examples of semi-arithmetic Fuchsian groups}

All Fuchsian triangle groups are semi-arithmetic. We further construct semi-arithmetic Fuchsian groups of signature $[0 ; 2,2,2, t ; 0]$ and of signature $[0 ; 2,2,2,2,2 ; 0]$ and show that there are infinitely many non-conjugate semiarithmetic Fuchsian groups of the same signature. 
Definition 5. Let $\Gamma$ be a cofinite Fuchsian group.

(i) The signature of $\Gamma$ is, as usual, described by $\left[g ; t_{1}, \ldots, t_{n} ; s\right]$ where $g$ is the genus, $n$ is the number of non-conjugate elliptic elements, $t_{i}, i=1, \ldots, n$, are the orders of these elements and $s$ is the number of non-conjugate cusps.

(ii) The signature of a Fuchsian triangle group is simply denoted by $[p, q, r]$; the signature of a Fuchsian quadrilateral group is simply denoted by $[p, q, r, t]$. (A Fuchsian quadrilateral group has genus zero and signature $[0 ; p, q, r, t ; 0]$ if $p, q, r, t$ are finite; if some of them are infinite, then they correspond to cusps.)

Lemma 2. Let $Q$ be a hyperbolic quadrilateral such that three of the interior angles are $\pi / 2$ while the fourth is $\pi / t$ for an integer $t>2$ or $t=\infty$. Let $E_{i}, i=1,2,3$, be the three vertices of $Q$ corresponding to angles $\pi / 2$, in the natural order. Let $\Gamma$ be a Fuchsian quadrilateral group of signature $[2,2,2, t]$ generated by elements $e_{i}$ of order 2 fixing $E_{i}, i=1,2,3$, and with a fundamental domain consisting of the quadrilateral $Q$ and a mirror image of $Q$ obtained by reflection in one side. Then the elements

$$
x:=e_{1} e_{2}, \quad y:=e_{2} e_{3}, \quad z:=e_{1} e_{3}
$$

of $\Gamma$ are hyperbolic and their respective axes contain the sides of $Q$ between $E_{1}$ and $E_{2}$, between $E_{2}$ and $E_{3}$ and the diagonal between $E_{1}$ and $E_{3}$. They satisfy

(i) $\left(\operatorname{tr}^{2}(x)-4\right)\left(\operatorname{tr}^{2}(y)-4\right)=16 \cos ^{2}(\pi / t)$;

(ii) $\operatorname{tr}^{2}(x) \operatorname{tr}^{2}(y)=4 \operatorname{tr}^{2}(z)$.

Proof. By construction, $x$ fixes the geodesic containing $E_{1}$ and $E_{2}$ etc. Recall that for all $E \in \mathrm{H}$ and all hyperbolic $\gamma \in \operatorname{PSL}(2, \mathrm{R})$ the hyperbolic distance $d$ satisfies

$$
\cosh \frac{1}{2} d(E, \gamma(E)) \geqslant \frac{1}{2}|\operatorname{tr}(\gamma)|
$$

with equality if and only if $E$ lies on the axis of $\gamma$. Using

$$
\cosh d\left(E_{1}, E_{2}\right)=\cosh \frac{1}{2} d\left(E_{1}, x\left(E_{1}\right)\right)=\frac{1}{2}|\operatorname{tr}(x)|
$$

and analogous formulae for $y$ and $z$ the lemma follows from well-known formulae in hyperbolic trigonometry; see for example Buser [3].

Lemma 3. Let $P$ be a hyperbolic right-angled pentagon. Let $E_{i}, i=1, \ldots, 5$, be the vertices of $P$ in the natural order. Let $\Gamma$ be the Fuchsian group of signature $[0 ; 2,2,2,2,2 ; 0]$ generated by elements $e_{i}$ of order 2 fixing $E_{i}, i=1, \ldots, 5$, and with a fundamental domain consisting of $P$ and a mirror image of $P$ obtained by reflection in one side. Then every $x_{i}:=e_{i} e_{i+1}$ (the index $i=1, \ldots, 5$, taken modulo 5) is a hyperbolic element of $\Gamma$ containing the side of $P$ between $E_{i}$ and $E_{i+1}$ on its axis. Every $z_{i}:=e_{i} e_{i+2}$, $i=1, \ldots, 5, i \bmod 5$, is hyperbolic as well and its axis contains the diagonal of $P$ between $E_{i}$ and $E_{i+2}$. Then, for $i=1, \ldots, 5$ (taking the indices modulo 5),

(i) $\left(\operatorname{tr}^{2}\left(x_{i}\right)-4\right)\left(\operatorname{tr}^{2}\left(x_{i+1}\right)-4\right)=4 \operatorname{tr}^{2}\left(x_{i+3}\right)$;

(ii) $\operatorname{tr}^{2}\left(x_{i}\right) \operatorname{tr}^{2}\left(x_{i+1}\right)=4 \operatorname{tr}^{2}\left(z_{i}\right)$.

Proof. This follows in the same way by well-known formulae in hyperbolic trigonometry, see again [3]. 
A proof of the following proposition can be found in Takeuchi [16].

Proposition 3. Let $\left\{a_{1}, a_{2}, \ldots, a_{n}\right\}$ be a set of generators of a cofinite Fuchsian group $\Gamma$. For any subset $\left\{i_{1}, \ldots, i_{m}\right\}$ of $\{1,2, \ldots, n\}$ let $t_{i_{1} \ldots i_{m}}:=\left|\operatorname{tr}\left(a_{i_{1}} \ldots a_{i_{m}}\right)\right|$. Then $\operatorname{Tr}(\Gamma)$ is contained in the ring

$$
\mathrm{Z}\left[t_{i_{1} \ldots i_{m}} \mid\left\{i_{1}, \ldots, i_{m}\right\} \subset\{1,2, \ldots, n\}\right] .
$$

COROLlary 2. Let $\Gamma$ be a Fuchsian quadrilateral group of signature $[2,2,2, t]$ as in Lemma 2. Let $x, y, z$, be defined as in Lemma 2. Then

$$
\operatorname{Tr}(\Gamma) \subset \mathrm{Z}\left[|\operatorname{tr}(x)|,|\operatorname{tr}(y)|,|\operatorname{tr}(z)|, 2 \cos \frac{\pi}{t}\right] .
$$

COROLlary 3. Let $\Gamma$ be a Fuchsian group of signature $[0 ; 2,2,2,2,2 ; 0]$ as in Lemma 3. Let $x_{i}, z_{i}, i=1, \ldots, 5$, be defined as in Lemma 3. Let $T=\left\{\left|\operatorname{tr}\left(x_{i}\right)\right|,\left|\operatorname{tr}\left(z_{i}\right)\right|\right.$ : $i=1, \ldots, 5\}$. Then

$$
\operatorname{Tr}(\Gamma) \subset \mathrm{Z}[T] .
$$

Proof. Here, $\Gamma$ is generated already by $e_{1}, \ldots, e_{4}$, say. Using the relations between all five generators it is easy to see that all traces of Proposition 3 can be expressed by elements of $T$.

We now introduce some terminology.

DeFINITION 6. Let $\Gamma$ be a cofinite Fuchsian group.

(i) Let $\Gamma$ be semi-arithmetic and let $\phi_{i}, i=1, \ldots, n$, be the $n$ embeddings of the corresponding number field $k=\mathrm{Q}\left(\operatorname{Tr}\left(\Gamma^{2}\right)\right)$ into $\mathrm{R}$. Then $\Gamma_{i}=\phi_{i}(\Gamma), i=1, \ldots, n$, is called an associated group.

(ii) A semi-arithmetic group $\Gamma$ is called stable if for all associated groups $\Gamma_{i}$ one of the two following statements hold:

(a) $\Gamma_{i}$ is conjugate in $\operatorname{PSL}(2, \mathrm{R})$ to $\Gamma$.

(b) The trace set of $\Gamma_{i}^{2}$ is contained in the interval $[-2,2]$.

We give some important consequences of these definitions.

Lemma 4. Let $\Gamma \subset \mathrm{SL}(2, \mathrm{R}), k$ and $\phi_{i}$ be as in Definition 6(i) and suppose that $\phi_{i}$ changes at most the signs of the traces $\operatorname{tr}(\gamma), \gamma \in \Gamma$, and so extends to a conjugation $\Gamma \longrightarrow \Gamma_{i}$ in $\operatorname{PSL}(2, \mathrm{R})$. Then $\phi_{i}=\mathrm{id}$ on $k$.

Proof. The embedding $\phi_{i}$ is the identity on $\operatorname{all}^{2}(\gamma), \gamma \in \Gamma$, generating the trace field $k$ of $\Gamma^{2}$ according to [15, Proposition 4].

Proposition 4. Arithmetic Fuchsian groups are stable.

Proof. By Takeuchi's definition of arithmetic Fuchsian groups (see Section 2) we have either $\operatorname{Tr}\left(\phi_{i}\left(\Gamma^{2}\right)\right) \subset[-2,2]$ or $\phi_{i}$ extends to the identity on $\Gamma$.

Proposition 5. Let $\Gamma$ be a Fuchsian triangle group. Then $\Gamma$ is semi-arithmetic. Moreover, $\Gamma$ is an arithmetic Fuchsian group if and only if $\Gamma$ is stable. 
Proof. Let $\Gamma$ have signature $[p, q, r]$. By Proposition $3, \operatorname{Tr}(\Gamma)$ is contained in $\mathrm{Z}[2 \cos (\pi / p), 2 \cos (\pi / q), 2 \cos (\pi / r)]$. It follows that $\Gamma$ is semi-arithmetic. The second part will follow from Proposition 6 in Section 4 and the fact that triangle groups admit modular embeddings.

REMARK 6. (i) The celebrated list of Takeuchi [16] of all arithmetic triangle groups can be obtained by applying Proposition 5 .

(ii) Proposition 5 is a particular case. We will show (and this is the main result of this section) that most of the stable semi-arithmetic Fuchsian groups are not arithmetic.

(iii) 'Most' of the semi-arithmetic Fuchsian groups are not stable.

Theorem 1. Let $t \in\{3,4,6, \infty\}$. Let $n$ be a positive integer with $n^{2} \geqslant 16 \cos ^{2}(\pi / t)$. Let $\Gamma(t, n)$ be a Fuchsian quadrilateral group of signature $[2,2,2, t]$ as in Lemma 2 . Let $x=x_{n}, y=y_{n}, z=z_{n}$ be defined as in Lemma 2. Let

\section{Then}

$$
\operatorname{tr}^{2}\left(x_{n}\right)=4+n-\sqrt{n^{2}-16 \cos ^{2}(\pi / t)} .
$$

(i) $\operatorname{tr}^{2}\left(y_{n}\right)=4+n+\sqrt{n^{2}-16 \cos ^{2}(\pi / t)}$;

(ii) $\operatorname{tr}^{2}\left(z_{n}\right)=2 n+4+4 \cos ^{2}(\pi / t)$;

(iii) $\Gamma(t, n)$ is semi-arithmetic and stable;

(iv) $\Gamma(t, n)$ is strictly semi-arithmetic, except in the cases

$$
(t, n) \in\{(3,2),(4,3),(6,4),(\infty, 4),(\infty, 5)\} \text {. }
$$

Proof. (i) and (ii) follow by Lemma 2. By construction and by Corollary 2, $\operatorname{Tr}(\Gamma(t, n))$ consists of integers of a totally real number field, and hence a fortiori also $\operatorname{Tr}\left(\Gamma^{2}(t, n)\right)$; therefore $\Gamma(t, n)$ is semi-arithmetic. Let $\phi$ be an embedding of $k=$ $\mathrm{Q}\left(\operatorname{Tr}\left(\Gamma^{2}(t, n)\right)\right)$ into $\mathrm{R}$ which is not the identity. The traces of the elliptic elements of order 2 remain invariant with respect to $\phi$ since $\phi$ maps an elliptic element to an elliptic element of the same order. In the case $t \in\{3,4,6, \infty\}$ it follows also that the absolute value of the trace of the elements of order $t$ cannot change. Under the action of $\phi$ the traces $\left|\operatorname{tr}\left(x_{n}\right)\right|$ and $\left|\operatorname{tr}\left(y_{n}\right)\right|$ are either fixed or exchanged. The first case would imply that $\phi \neq$ id extends to a conjugation of $\Gamma$ in $\operatorname{PSL}(2, R)$ which is impossible according to Lemma 4 . In the second case, the Fuchsian group $\phi(\Gamma(t, n))$ has a fundamental domain of the same geometry (the quadrilateral $Q$ may be replaced by a mirror image in one side) and hence is conjugate in $\operatorname{PSL}(2, \mathrm{R})$ to $\Gamma(t, n)$. Therefore, $\Gamma(t, n)$ is stable.

(iv) By the trace identity

$$
\operatorname{tr}\left(x^{2}\right)=\operatorname{tr}^{2}(x)-2
$$

we have $\operatorname{tr}\left(x_{n}^{2}\right)=2+n-\sqrt{n^{2}-16 \cos ^{2}(\pi / t)}$. If $\Gamma$ is an arithmetic Fuchsian group, then by the arguments used in the proof of Proposition 4 there cannot exist a non-trivial embedding $\phi$ of $k$ whose extension sends $\Gamma$ to a Fuchsian group; therefore $\sqrt{n^{2}-16 \cos ^{2}(\pi / t)}$ must be a rational integer. This is only true in the cases mentioned. Moreover, it is easy to see that in these cases $\Gamma(t, n)$ is an arithmetic Fuchsian group.

REMARK 7. There exists only a finite number of mutually non-conjugate arithmetic Fuchsian groups of a fixed signature. Theorem 1 shows that an infinite 
number of different stable semi-arithmetic Fuchsian groups can exist for a given signature. This shows that most of the semi-arithmetic Fuchsian groups are strictly semi-arithmetic. It is important to emphasize that in the strictly semi-arithmetic cases the embedding $\phi$ with

$$
\phi\left(\sqrt{n^{2}-16 \cos ^{2}(\pi / t)}\right)=-\sqrt{n^{2}-16 \cos ^{2}(\pi / t)}
$$

changes not only the signs of the traces. Thus its extension maps the group $\Gamma(t, n)$ to the conjugated Fuchsian group $\phi(\Gamma(t, n))$ but without $\phi$ being a conjugation in $\operatorname{PSL}(2, R)$. The same remark applies to the following theorem.

THEOREM 2. Let $n \geqslant 6$ be an integer. Let $\Gamma(n)$ be a Fuchsian group of signature $[0 ; 2,2,2,2,2 ; 0]$ as in Lemma 3. Let $x_{i}=x_{i}(n), z_{i}=z_{i}(n), i=1, \ldots, 5$, be defined as in Lemma 3. For all $n$ let $\operatorname{tr}^{2}\left(x_{4}\right)=8$. Let

Then

$$
\operatorname{tr}^{2}\left(x_{1}(n)\right)=4+n-\sqrt{n^{2}-32} .
$$

(i) $\operatorname{tr}^{2}\left(x_{2}(n)\right)=4+n+\sqrt{n^{2}-32}$,

$\operatorname{tr}^{2}\left(x_{3}(n)\right)=8+n-\sqrt{n^{2}-32}$,

$\operatorname{tr}^{2}\left(x_{5}(n)\right)=8+n+\sqrt{n^{2}-32}$,

$\operatorname{tr}^{2}\left(z_{1}(n)\right)=12+2 n$,

$\operatorname{tr}^{2}\left(z_{2}(n)\right)=16+3 n+\sqrt{n^{2}-32}$,

$\operatorname{tr}^{2}\left(z_{3}(n)\right)=2 \operatorname{tr}^{2}\left(x_{3}(n)\right)$,

$\operatorname{tr}^{2}\left(z_{4}(n)\right)=2 \operatorname{tr}^{2}\left(x_{5}(n)\right)$,

$\operatorname{tr}^{2}\left(z_{5}(n)\right)=16+3 n-\sqrt{n^{2}-32}$

(ii) $\Gamma(n)$ is semi-arithmetic and stable;

(iii) $\Gamma(n)$ is strictly semi-arithmetic except in the cases $n=6$ and $n=9$.

Proof. Part (i) follows by a calculation applying Lemma 3. In analogy to Theorem 1, part (ii) is a consequence of (i), Lemma 4 and Corollary 3. Concerning (iii) we have $\operatorname{tr}^{2}\left(x_{1}(n)\right)-2=2+n-\sqrt{n^{2}-32}$. If $\Gamma(n)$ is an arithmetic Fuchsian group, then $\sqrt{n^{2}-32}$ must be rational which is only the case if $n=6$ or $n=9$. Moreover, it is easy to see that in these cases $\Gamma(n)$ is an arithmetic group.

REMARK 8. The Fuchsian groups of signature $[0 ; 2,2,2,2,2 ; 0]$ in Theorem 2 and the quadrilateral groups of signature $[2,2,2, t], t \in\{3,4,6\}$, in Theorem 1 both have torsion-free cocompact subgroups which give closed Riemann surfaces; see the next lemma. Some of these subgroups are stable too. As in the above-treated cases there then exists an infinite family of stable semi-arithmetic groups of the same signature which shows that this phenomenon also exists for universal covering groups of closed surfaces.

\section{Modular embeddings}

Lemma 5. Let $\Gamma$ be a semi-arithmetic group.

(i) There is a torsion-free subgroup $\Gamma^{\prime}$ of finite index in $\Gamma$.

(ii) Suppose that $\Gamma^{\prime}$ is a torsion-free subgroup of $\Gamma$ of finite index and that the embedding

$$
\phi: \mathrm{Q}\left(\operatorname{Tr}\left(\Gamma^{2}\right)\right) \longrightarrow \mathrm{R}
$$

extends to a conjugation $\phi \mid \Gamma^{\prime}$ in $\operatorname{PSL}(2, \mathrm{R})$. Then $\phi$ extends also to a conjugation of $\Gamma$ in $\operatorname{PSL}(2, \mathrm{R})$. 
Proof. (i) is a special case of a result of Fox [6] and Mennicke [10].

(ii) Assume that $\phi \mid \Gamma^{\prime}$ is a conjugation in $\operatorname{PSL}(2, R)$. Since for every hyperbolic element $\gamma \in \Gamma$ some power $\gamma^{m}$ is contained in $\Gamma^{\prime}$ and since the hyperbolic elements generate $\Gamma$, it follows that the conjugation $\phi \mid \Gamma^{\prime}$ extends to a conjugation $\phi \mid \Gamma$.

Proposition 6. A stable semi-arithmetic Fuchsian group $\Gamma$ admits a modular embedding if and only if $\Gamma$ is an arithmetic Fuchsian group.

Proof. It is clear that every arithmetic Fuchsian group admits a modular embedding. Assume now that $\Gamma$ is stable semi-arithmetic, but not arithmetic. Then there exists an embedding $\phi_{i}$ of $k=\mathrm{Q}\left(\operatorname{Tr}\left(\Gamma^{2}\right)\right)$ into $\mathrm{R}$ such that the associated group $\Gamma_{i}$ is conjugate in $\operatorname{PSL}(2, \mathrm{R})$ to $\Gamma$ and such that $\phi_{i} \mid \Gamma$ is still not a conjugation in $\operatorname{PSL}(2, R)$. By Lemma $5, \phi_{i}$ is not a conjugation if restricted to the hyperbolic elements of $\Gamma$. Assume now that $\Gamma$ admits a modular embedding $(f, F)$. Then by Proposition 2 the functional equation

$$
F_{i}(\gamma z)=\phi_{i}(\gamma) F_{i}(z)
$$

for every $z \in \mathrm{H}$ and every $\gamma \in \Gamma$ is solvable by a holomorphic function $F_{i}: \mathrm{H} \longrightarrow \mathrm{H}$ which cannot be given by a fractional linear transformation. Choose a $z$ on the axis of a hyperbolic $\gamma \in \Gamma$ and compare the hyperbolic distances between $z$ and $\gamma(z)$ and between their $F_{i}$-images; it follows by the Schwarz-Pick lemma that $\left|\operatorname{tr}\left(\phi_{i}(\gamma)\right)\right|$ is strictly smaller than $|\operatorname{tr}(\gamma)|$ for every hyperbolic element $\gamma \in \Gamma$, which contradicts the facts that $\Gamma_{i}$ is conjugate to $\Gamma$ and that $\Gamma$ has a hyperbolic element with minimal $|\operatorname{tr}(\gamma)|$.

COROLlaRY 4. The strictly semi-arithmetic Fuchsian groups constructed in Theorems 1 and 2 do not admit a modular embedding.

Proposition 6 can be generalized in the following way.

THEOREM 3. Let $\Gamma$ be a semi-arithmetic group admitting a modular embedding. Then for any embedding

$$
\phi: \mathrm{Q}\left(\operatorname{Tr}\left(\Gamma^{2}\right)\right) \longrightarrow \mathrm{R}
$$

we have the following alternatives:

(i) $\phi=$ id;

(ii) $\phi(\Gamma)$ is not a Fuchsian group.

Proof. Suppose $\Gamma$ to be semi-arithmetic and

$$
\phi: \mathrm{Q}(\operatorname{Tr}(\Gamma)) \longrightarrow \mathrm{R}
$$

to be a non-trivial embedding, which is by Lemma 4 equivalent to the hypothesis that the extension of $\phi$ to $\Gamma$ does not induce a conjugation in $\operatorname{PSL}(2, R)$. Now assume that $\phi(\Gamma)$ is a Fuchsian group; this assumption will lead to a contradiction.

(i) By Lemma 5, we may assume that $\Gamma$ is torsion-free. Now $\phi(\Gamma)$ cannot be conjugate to $\Gamma$ by the argument used in the proof of Proposition 6 for stable semiarithmetic groups admitting a modular embedding. Assume first that $\Gamma$ is cocompact. Let $(f, F)$ be a modular embedding of $\Gamma$. By Proposition 2, the component $F_{j}$ of $F$ corresponding to $\phi$ induces then a holomorphic map

$$
\hat{F}_{j}: \mathrm{H} / \Gamma \longrightarrow \mathrm{H} / \phi(\Gamma)
$$

of the corresponding Riemann surfaces which are non-isomorphic since $\Gamma$ and $\phi(\Gamma)$ are not conjugate. Generators and relations show that $\phi(\Gamma)$ is cocompact 
also, and that $\mathrm{H} / \Gamma$ and $\mathrm{H} / \phi(\Gamma)$ have the same genus (>1) which implies that $\hat{F}_{j}$ is constant. Then $F_{j}$ also would be constant, contradicting the functional equation of Proposition 2.

(ii) The same argument extends to the case where $\Gamma$ is non-cocompact. Passing to a subgroup of finite index if necessary we may still suppose that the genus of $\Gamma$ and $\phi(\Gamma)$ is $>1$. Then $\hat{F}_{j}$ extends to the compactified quotient space for the following reason. If $\gamma \in \Gamma$ generates a parabolic subgroup of $\Gamma$, the same is true for $\phi(\gamma)$ in $\phi(\Gamma)$. We can suppose that both $\gamma$ and $\phi(\gamma)$ act on $\mathrm{H}$ as $z \longmapsto z+1$. Using the local coordinate $q:=e^{2 \pi i z}$, the functional equation shows that $F_{j}$ induces a holomorphic mapping of the pointed unit disk

$$
\{q: 0<|q|<1\}
$$

into itself, which is extendable by Riemann's theorem on removable singularities to the unit disk; $\hat{F}_{j}$ is hence extended to the cusps.

We can give a reformulation of Theorem 3 and of the arguments used in the proof of Proposition 6 in a more geometric language.

COROLlary 5. Assume that a cofinite Fuchsian group $\Gamma$ admits a modular embedding. Then for all non-trivial embeddings

$$
\mathrm{id} \neq \phi: \mathrm{Q}\left(\operatorname{Tr}\left(\Gamma^{2}\right)\right) \longrightarrow \mathrm{R}
$$

the point set $\phi(\operatorname{Tr}(\Gamma))$ is non-discrete and

$$
|\operatorname{tr}(\phi(\gamma))|<|\operatorname{tr}(\gamma)|
$$

for every hyperbolic element $\gamma \in \Gamma$.

We can also give a reformulation in the language of lattices.

COROllary 6. Assume that a Fuchsian group $\Gamma$ admits a modular embedding. Let $k=\mathrm{Q}\left(\operatorname{Tr}\left(\Gamma^{2}\right)\right)$ be of degree $n$ over $\mathrm{Q}$ and let $\mathcal{O}$ be the ring of integers of $k$. Represent (1) as usual by a lattice in $\mathrm{R}^{n}$. Let $\operatorname{Tr}\left(\Gamma^{2}\right)$ be represented as a subset of this lattice. Then among the projections of $\operatorname{Tr}\left(\Gamma^{2}\right)$ to the coordinate axes, there is exactly one with a discrete image. Moreover, $\operatorname{Tr}\left(\Gamma^{2}\right)$ is contained in the cone

$$
\left\{\left(x_{1}, \ldots, x_{n}\right) \in \mathrm{R}^{n}:\left|x_{i}\right| \leqslant\left|x_{1}\right| \text { for all } i=2, \ldots, n\right\} .
$$

The results of this paper raise the following problem.

Problem 1. Assume that a Fuchsian group $\Gamma$ admits a modular embedding. Is $\Gamma$ arithmetic or a subgroup of a Fuchsian triangle group?

REMARK 9. (i) In order to treat this problem it seems that much more should be known on behalf of the non-discrete subgroups of $\operatorname{PSL}(2, \mathrm{R})$; for a recent reference concerning this subject see Beardon [1].

(ii) Using rigidity arguments and the fact that $\mathrm{H}^{r} / \Delta$ (compactified if necessary) can be considered as an algebraic variety defined over some number field, one can even prove a further restriction for Fuchsian groups $\Gamma$ admitting modular embeddings: their Riemann surfaces $\mathrm{H} / \Gamma$ (compactified if necessary) can also be considered as algebraic curves defined over a number field. Therefore a positive answer 
to the question above would follow from a much more general conjecture of Chudnovsky and Chudnovsky [4, Section 7] about arithmetic aspects of the uniformization of algebraic curves and their relations to Fuchsian differential equations. In particular, according to [4], curves defined over number fields should have universal covering groups $\Gamma \subset \operatorname{PSL}(2, k), k$ a number field, only if $\Gamma$ is an arithmetic Fuchsian group or a subgroup of a Fuchsian triangle group.

Acknowledgements. We thank the referee for valuable comments and for providing us with useful additional references. The first author was partly supported by the Schweiz. Nationalfonds during the work on this paper. The second author thanks the Kyushu University, Fukuoka, and the Chiba University for their kind hospitality in September 1996 when parts of a first version of this paper were written.

\section{References}

1. A. F. Beardon, 'Some remarks on non-discrete Möbius groups', Ann. Acad. Sci. Fenn. Math. 21 (1996) 69-79.

2. A. Borel, 'Commensurability classes and volumes of hyperbolic 3-manifolds', Ann. Scuola Norm. Sup. Pisa (Ser. IV) 8 (1981) 1-33.

3. P. BUSER, Geometry and spectra for compact Riemann surfaces (Birkhäuser, Boston, 1992).

4. D. V. Chudnovsky and G. V. Chudnovsky, 'Computer algebra in the service of mathematical physics and number theory', Computers and Mathematics. Proceedings of International Conference, Stanford, 1986, Lecture Notes in Pure and Applied Mathematics 125 (Dekker, 1990) 109-232.

5. P. COHEN and J. Wolfart, 'Modular embeddings for some non-arithmetic Fuchsian groups', Acta Arith. 61 (1990) 93-110.

6. R. H. Fox, 'On Fenchel's conjecture about F-groups', Mat. Tidsskr. B (1952) 61-65.

7. H. M. Hilden, M. T. Lozano and J. M. Montesinos-Amilibia, 'A characterization of arithmetic subgroups of $S L(2, \mathrm{R})$ and $S L(2, \mathrm{C})$ ', Math. Nachr. 159 (1992) 245-270.

8. S. KatoK, Fuchsian groups (University of Chicago Press, Chicago, 1992).

9. W. Luo and P. SARnaK, 'Number variance for arithmetic hyperbolic surfaces', Comm. Math. Phys. 161 (1994) 419-432.

10. J. Mennicke, ‘Eine Bemerkung über Fuchssche Gruppen', Invent. Math. 2 (1967) 301-305; 6 (1968) 106.

11. A. W. ReID, 'A note on trace-fields of Kleinian groups', Bull. London Math. Soc. 22 (1990) 349-352.

12. T. A. Schmidt, 'Klein's cubic surface and a "non-arithmetic" curve', Math. Ann. 309 (1997) 533-539.

13. P. Schmutz, 'Arithmetic groups and the length spectrum of Riemann surfaces', Duke Math. J. 84 (1996) 199-215.

14. G. Shimura, 'Construction of class fields and zeta functions of algebraic curves', Ann. Math. 85 (1967) $58-159$.

15. K. TAKEUChI, 'A characterization of arithmetic Fuchsian groups', J. Math. Soc. Japan 27 (1975) $600-612$.

16. K. TaKeuchi, 'Arithmetic triangle groups', J. Math. Soc. Japan 29 (1977) 91-106.

17. P. L. Waterman and C. MaClachlan, 'Fuchsian groups and algebraic number fields', Trans. Amer. Math. Soc. 287 (1985) 353-364.

Section de Mathématiques

Université de Genève

Case Postale 240

CH-1211 Genève 24

Switzerland

Paul.Schmutz@math.unige.ch

\author{
Mathematisches Seminar \\ Goethe Universität \\ Robert Mayer-Straße 6-10 \\ D-60054 Frankfurt-am-Main \\ Germany
}

wolfart@math.uni-frankfurt.de 